\section{Making vaccines is no yolk}

\section{By Tim Fulmer, Senior Writer}

Conventional production of influenza vaccine in eggs has multiple limitations, which has spurred work on cell-based approaches. iBio Inc. and the Fraunhofer USA Center for Molecular Biotechnology have been working on a third approach-plant-based vaccine production-and in September took an H1N1 influenza vaccine generated using their process into a Phase I trial.

The partners believe the platform could generate vaccine more cheaply and rapidly than the traditional egg-based method of flu vaccine production while requiring less investment in manufacturing infrastructure than cell culture approaches.

Influenza vaccine production still relies on a 50-year-old method that involves growing the flu virus in hens' eggs, breaking the eggs open and purifying the vaccine antigens. But the pro-
"We believe speed of production and a significant reduction in cost are the key advantages of our approach over the eggbased approach." -Robert Erwin, iBio Inc.
Robert Erwin, president of iBio. "We believe speed of production and a significant reduction in cost are the key advantages of our approach over the egg-based approach."

The plant-based approach could also offer advantages over cell culture methods. "By our estimate, the infrastructure cost for working with plants is about one-eighth what it is for cell culture-based methods," said Vidadi Yusibov, executive director of Fraunhofer USA and CSO of iBio. "Due to simplicity and cost, it could be easier to establish plant-based manufacturing facilities in various geographic locations than to build the complex facilities required for large-scale cell culture-based production."

The most advanced cell culture-based method for influenza vaccine production is being developed by Protein Sciences Corp. The company's FluBlok, a trivalent seasonal influenza vaccine made with recombinant antigens purified from cultured insect cells, is under FDA review. If approved, it would be the first cell culture-derived influenza vaccine licensed in the U.S.

According to Manon Cox, CEO of Protein Sciences, "by our estimate, 30-40 days would be necessary from the time a pandemic flu virus is identified to the first production of a FluBlok vaccine."

Daniel Adams, executive chairman of Protein Sciences, thinks that plant-based vaccine production could face regulatory challenges. "Regulators will want proof there is minimal variability from plant to plant in terms of the quality of the vaccess has numerous drawbacks. ${ }^{1}$ For example, vaccine manufacturers are dependent on a supply of chicken eggs that is difficult to increase quickly, creating long lead times for new vaccine production.

Also, adapting flu virus to grow in eggs can potentially select for strains that are not matched with the circulating flu strain, leading to a less effective vaccine. Finally, there is the potential for allergic reactions to egg components.

At least 10 companies thus have opted to avoid eggs altogether by growing vaccine virus in cultured cells. ${ }^{2}$

iBio and Fraunhofer have taken a third route: plants. Their method uses a combination of genetically engineered plant-targeting bacteria and genetically unmodified plants. Fraunhofer, a not-for-profit organization, developed the plant-based method and in 2004 transferred ownership of the IP to iBio.

The method begins with a viral vector expressing the influenza hemagglutinin antigen that is introduced into a plant-targeting bacterium. Tobacco plants are then dipped into a solution of the bacteria, which rapidly and uniformly infiltrate the plant tissue under a vacuum. Once inside the plant, the bacteria multiply, expressing high levels of the vaccine antigen. Within a few weeks the plants are harvested and the antigen is purified from the plant biomass.

"Unlike the egg-based method, which could take up to six months for turnaround in times of a pandemic, we believe our plant-based approach could take only several weeks to produce new vaccine," said cine antigen produced," he said. "Among other things, that means proving that the plants aren't susceptible to viral infections, as well as showing that the downstream processing and purification steps don't carry through plant-derived compounds that are immunogenic."

In contrast, Adams said Protein Sciences' approach "uses a single insect cell line, which we have proven does not harbor any extraneous viruses. With a single source of homogeneous material, we can be sure we've minimized variability throughout the manufacturing process."

According to Yusibov, the plant-based production process has been developed "to remove plant and human viruses as well as bacteria. There are no products of animal origin used in our manufacturing process, which is refined to remove all host proteins and small molecules, including ones that could potentially cause an allergic reaction."

Moreover, iBio uses indoor hydroponics facilities to grow plant biomass to avoid potential variation caused by soil and climate conditions, Yusibov added.

Medicago Inc. is working on a similar platform that could directly compete with iBio's approach, also using plant-targeting bacteria to generate production of the hemagglutinin antigen in tobacco plants. However, rather than using a viral vector to express the recombinant antigen, Medicago uses noninfectious virus-like particles (VLPs), which have a structure that mimics native virus. ${ }^{3}$

"Our VLPs express and display the full hemagglutinin protein, which has more epitopes and a potentially broader reactivity than 
only the globular domain of hemagglutinin, which is the antigen expressed by viral vector systems like iBio's," said Nathalie Charland, director of product portfolio at Medicago.

The company's $\mathrm{H} 5 \mathrm{~N} 1$ vaccine produced using the plant-based method has completed a Phase I trial and is set to begin Phase II testing next month. The company also has vaccines against $\mathrm{H} 1 \mathrm{~N} 1$ and seasonal flu in preclinical development and plans to begin a Phase I/II trial of the H1N1 vaccine in 1Q11.

Charland told SciBX that Medicago showed proof of principle of its method's speed during last year's H1N1 outbreak. "We required only 19 days from the time the genetic sequence of the H1N1 strain was made public to first production of vaccine," she said.

Erwin said iBio and its Fraunhofer collaborators are also using their platform to generate vaccines beyond H1N1. "With support from the U.S. Department of Defense and the Bill \& Melinda Gates Foundation, we have preclinical programs to develop vaccines against malaria, sleeping sickness and plague/anthrax," he said.

Fulmer, T. SciBX 3(38); doi:10.1038/scibx.2010.1141

Published online Sept. 30, 2010

\section{REFERENCES}

1. Ulmer, J.B. et al. Nat. Biotechnol. 11, 1377-1383 (2006)

2. Usdin, S. \& McCallister, E. BioCentury 17(20), A1; May 4, 2009

3. D'Aoust, M.-A. et al. Plant Biotechnol. J. 8, 607-619 (2010)

COMPANIES AND INSTITUTIONS MENTIONED

Bill \& Melinda Gates Foundation, Seattle, Wash.

iBio Inc. (OTCBB:IBPM), Newark, Del.

Fraunhofer USA Center for Molecular Biotechnology, Newark, Del.

Medicago Inc. (TSX:MDG), Quebec City, Quebec, Canada

Protein Sciences Corp., Meriden, Conn.

U.S. Department of Defense, Washington, D.C. 